\title{
Socially Anxious Tendencies Affect Autonomic Responses during Eye Gaze Perception
}

\author{
Yuki Tsuji ${ }^{1}$, Sotaro Shimada ${ }^{2 *}$ \\ ${ }^{1}$ Department of Electrical Engineering, Graduate School of Science and Technology, Meiji University, \\ Kawasaki, Japan \\ ${ }^{2}$ Department of Electronics and Bioinformatics, School of Science and Technology, Meiji University, \\ Kawasaki, Japan \\ Email: tsuji@meiji.ac.jp, ${ }^{*}$ sshimada@meiji.ac.jp
}

Received 7 September 2015; accepted 12 October 2015; published 15 October 2015

Copyright (c) 2015 by authors and Scientific Research Publishing Inc.

This work is licensed under the Creative Commons Attribution International License (CC BY).

http://creativecommons.org/licenses/by/4.0/

(c) (7)

Open Access

\begin{abstract}
Social anxiety disorder is a common psychiatric disorder. The gaze of others is known to frequently induce social anxiety. We conducted a gaze detection experiment to examine the effects of social anxiety on autonomic response, namely heart rate (HR) response. We used the maximum HR deceleration between $0 \mathrm{~s}$ and $3 \mathrm{~s}$ after stimulus onset as an indicator of emotional stress. Participants were assigned to a high social anxiety (HSA) or low social anxiety (LSA) group on the basis of cut-off scores indicative of clinical levels of stress as per the Japanese version of the Liebowitz Social Anxiety Scale (LSAS-J). Our results showed that HR deceleration was greater for the HSA compared with the LSA group. Moreover, the higher the LSAS-J score was, the greater the increase was in HR deceleration (correlation coefficient $r_{s}=0.52, p<0.01$ ). Our results suggest that the eye gaze of others can be processed as a threat in individuals with a high tendency towards social anxiety.
\end{abstract}

\section{Keywords}

Social Anxiety Disorder, Gaze Perception, Electrocardiogram (ECG), Heart Rate Deceleration

\section{Introduction}

Information obtained via eye gaze is important in human social interactions and communication. Eye gaze ${ }^{*}$ Corresponding author. 
serves as a social signal that conveys the direction of an individual's attention and interest (Baron-Cohen, 1995). Individuals with social anxiety disorders (SAD) tend to perceive this signal as a threat and often seek to avoid the eye gaze of others (Greist, 1995). SAD is one of the most common psychiatric disorders with a lifetime prevalence and lifetime morbid risk of $10.7 \%$ and $13.0 \%$, respectively (Kessler, Petukhova, Sampson, Zaslavsky, \& Wittchen, 2012). SAD and major depressive disorder (MDD) often occurs together, and SAD precedes MDD in approximately 70\% of patients (Kessler, Stang, Wittchen, Stein, \& Walters, 1999).

Avoidance or excessive fear of situations associated with being evaluated or embarrassed by others is a defining feature of SAD or social phobia (American Psychiatric Association, 2013). Particularly, the gaze of others is known to frequently induce social anxiety. Recent studies have shown that individuals with SAD avoid the gaze of others, which is perceived as threatening (Horley, Williams, Gonsalvez, \& Gordon, 2003; Moukheiber et al., 2010; Weeks, Howell, \& Goldin, 2013) and that such self-directed gaze may elicit fears of being stared at (Schneier, Rodebaugh, Blanco, Lewin, \& Liebowitz, 2011).

There are two main types of defense strategies in response to a threat: active defense and passive defense. Fight/flight behavior is an active defense strategy that is often used when it is possible to escape from a threat (Cannon, 1915). The autonomic changes associated with active defense strategies are mediated predominantly by sympathetic activation, including hypertension, tachycardia, and temporary heart rate (HR) increase (HR acceleration). Passive defense strategies, such as immobilization or freezing behavior, are usually elicited when threat is inescapable, and are mediated predominantly by parasympathetic activation, including hypotension, bradycardia, and temporary HR reduction (HR deceleration) (Henry \& Stephens, 1977). HR deceleration, which is often related to freezing behavior or immobility, has been used as a main outcome measure for fear in animal studies. Hermans, Henckens, Roelofs, \& Fernández (2012) showed that neural activity in the amygdala-periaqueductal grey pathway can be linked to freezing behavior through an association with parasympathetic as opposed to sympathetic dominated autonomic responses. Bradley, Codispoti, Cuthbert, \& Lang (2001) observed more pronounced HR deceleration when healthy individuals were confronted with unpleasant images (e.g. accidents and violence) from the International Affective Picture System (IAPS), compared with pleasant or neutral pictures. However, whether HR is modulated by eye gaze perception in individuals with SAD has not been fully investigated (Wieser, Pauli, Alpers, \& Mühlberger, 2009).

The purpose of this study was to examine the effects of socially anxious tendencies on HR responses during a gaze detection experiment. We hypothesized that HR deceleration during eye gaze perception would correspond with the degree of SAD tendencies, reflecting a potential fearful response to the gaze of others.

\section{Methods}

\subsection{Participants}

Fifteen healthy volunteers (3 women, 12 men, $22.5 \pm 3.4$, mean \pm SD) took part in this experiment. All participants had normal or corrected to normal vision and gave written informed consent to participate in the study. We measured social anxiety in the participants by means of the Japanese version of the Liebowitz Social Anxiety Scale (LSAS-J) (Liebowitz, 1987). Participants also completed the Beck Depression Inventory-II (BDI-II) (Beck, Steer, Ball, \& Ranieri, 1996). The experiments were approved by the ethics committee of the School of Science and Technology, Meiji University, and were conducted according to the principles and guidelines of the Declaration of Helsinki.

\subsection{Questionnaire}

We used the LSAS-J to assign subjects to high (HSA) and low social anxiety (LSA) groups. The LSAS-J is a 24-item scale that measures fear and avoidance of social situations over the past week. It is composed of 11 questions related to social interaction situations (e.g. going to a party, meeting strangers) and 13 questions concerning public performance (e.g. public speaking, eating in public places). The first rating is a measure of fear/anxiety and the second rating is a measure of avoidance. Participants responded using a 4-point (0 - 3) Likert-type scale. The total score of the LSAS-J ranges from 0 - 144. We measured levels of depression using the Beck Depression Inventory-II (BDI-II), which consisted of 21 questionnaire items. The total score of the BDI-II ranges from 0 to 63 . 


\subsection{Stimuli \& Procedure}

Stimuli were presented on the center of a 27 inch LCD monitor using E-Prime software (Psychology Software Tools, USA). The stimuli were viewed from a distance of approximately $100 \mathrm{~cm}$, and occupied $11.8^{\circ} \times 26.0^{\circ}$ in the visual field $(20.5 \mathrm{~cm} \times 46.0 \mathrm{~cm})$. Each experimental stimulus consisted of eight pairs of eyes with varying gaze directions arranged in a circle (Figure 1). The eyes were gray scale images of eyes engaged in either a direct or averted eye gaze (leftward-gaze or rightward-gaze). In the target present condition, the stimuli consisted of a target (leftward or rightward averted gaze), opposite-directed averted gazes, and direct gazes (Figure 1). In the target absent condition, the stimuli consisted of averted gazes that were opposite-directed with respect to the target and direct gazes (Figure 1). We varied the number of pairs of eyes engaged in a direct gaze, such that there were one, three, five, or seven pairs. The stimuli were produced from images of one of the experimenters, using Adobe Photoshop 6.0 software.

The experiment consisted of two factors: the presence of the target (present or absent) and the number of eye pairs engaged in a direct eye gaze $(1,3,5$, or 7$)$. The participants underwent 8 trials in each condition, for a total of 64 trials. In each trial, the experimental stimulus was displayed for $10 \mathrm{~s}$, and then a fixation cross appeared for $20 \mathrm{~s}$. The participant was required to detect eye pairs engaged in a leftward or rightward averted gaze (target) among opposite-directed averted and direct gazes. The participants were required to press one of two buttons according to whether the target (leftward-gaze or rightward-gaze) was present or absent in each trial. We measured reaction times (RTs) according to button press responses. The direction of the target gaze (leftward or rightward) and the number of direct gazes were counterbalanced among participants.

\subsection{ECG Recording}

We measured electrocardiogram (ECG) data from lead II using a bio-signal amplifier (g.USBamp; g.tec Inc., Schiedlberg, Austria). To calculate instantaneous HR in beats per minute (bpm), we detected the interval between R-waves, the most prominent peaks of the ECG. We defined HR deceleration for each participant and each trial as an evoked negative HR peak between $0 \mathrm{~s}$ and $3 \mathrm{~s}$ after the stimulus onset. We defined HR acceleration as an evoked positive HR peak between $3 \mathrm{~s}$ and $6 \mathrm{~s}$ after the stimulus onset (Bradley, Codispoti, Cuthbert, \& Lang, 2001).

\subsection{Statistical Analysis}

The mean RTs, the mean HR deceleration, and the mean HR acceleration were separately submitted to 3-way repeated measures analysis of variance (ANOVA). We used the number of direct gaze eye pairs $(1,3,5$, or 7$)$ and the presence of the target stimulus (present or absent) as within-subject factors, and the participant group (LSA or HSA) as a between-subject factor. We used the Spearman's rank correlation $\left(r_{s}\right)$ to examine the correlations between LSAS-J scores and RTs and between LSAS-J scores and HR deceleration.

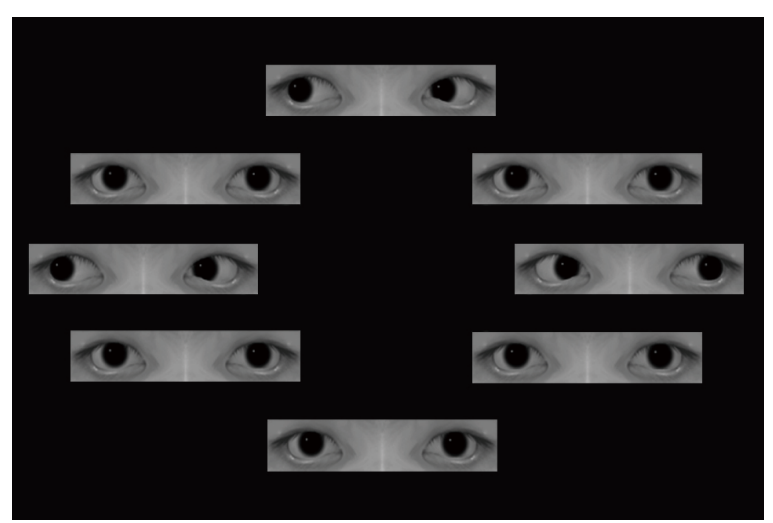

(a)

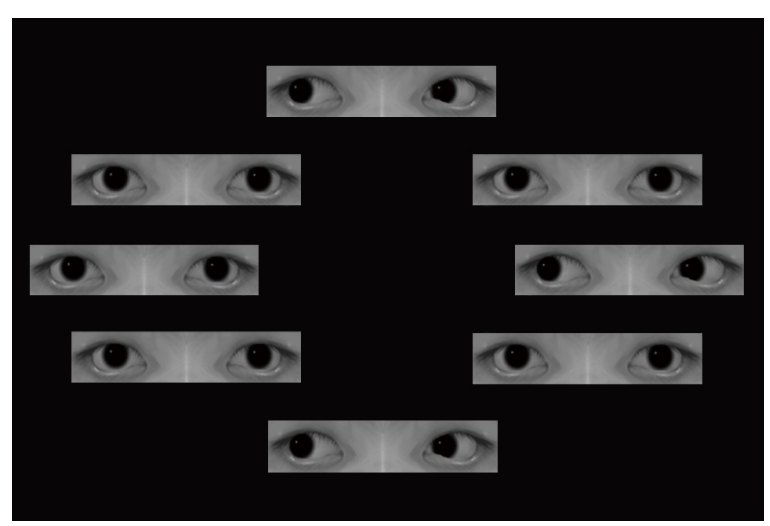

(b)

Figure 1. Examples of the stimulus display in the experiment. (a) The target (rightward-gaze, appeared to the right position of the stimulus array) present condition, in which the target is presented among distracters (leftward-gaze and direct gaze). (b) The target (rightward-gaze) absent condition, in which the target is absent. 


\section{Results}

We divided the participants into two groups, those with high social anxiety (HSA, n = 7) and those with low social anxiety (LSA, $n=8$ ), on the basis of the cut-off point at which SAD is probable (LSAS-J score of 60, see Table 1). The LSAS-J scores of the two groups were significantly different (Wilcoxon's Rank Sum Test, $p<$ 0.01).

\subsection{Behavioral Result}

We found that the presence of the target had a significant effect on RTs $\left(F(1,13)=75.6, p<0.01, \eta^{2}=0.29\right)$. Specifically, RTs in the absent condition were longer than those in the present condition. We did not find any other significant main effects or interactions $(p>0.10)$. We found no significant correlation between LSAS-J and RTs $\left(r_{s}=0.21, p>0.10\right)$.

\subsection{Heart Rate}

We observed a peak in HR deceleration during the 0 - 3 s period after stimulus onset and a peak in HR acceleration during the 3 - 6 s period following stimulus onset each group (Figure 2).

For HR acceleration, we found no significant main effects or interactions. For HR deceleration, we found a significant main effect of group $\left(F(1,13)=9.9, p<0.01, \eta^{2}=0.17\right)$, indicating that the HR deceleration in the HSA group was greater than that in the LSA group (Figure 2). We did not find any other significant main effects or interactions.

We did not find any significant correlation between LSAS-J scores and HR acceleration $\left(r_{s}=0.02, p>0.10\right)$. However, a positive correlation between LSAS-J score and HR deceleration was significant $\left(r_{s}=0.52, p<0.05\right.$, Figure 3). This result indicates that the higher the LSAS-J score, the greater the increase in HR deceleration. In

Table 1. Participant characteristics.

\begin{tabular}{lcccc}
\hline & LSA $(\mathrm{n}=8)$ & HSA $(\mathrm{n}=7)$ & $Z$ & $p$ \\
\hline & M (SD) & M (SD) & & $<0.01$ \\
LSAS-J & $32.5(14.1)$ & $71.7(13.2)$ & -3.19 & $<0.01$ \\
-Social interactions & $15.8(6.86)$ & $36.4(8.38)$ & -2.95 & $<.01$ \\
-Performance situations & $16.8(8.38)$ & $35.3(6.58)$ & -3.19 & $>0.20$ \\
BDI-II & $5.63(6.02)$ & $9.29(3.90)$ & -1.28 & $>0.28$ \\
\hline
\end{tabular}

M, mean; SD, standard deviation.

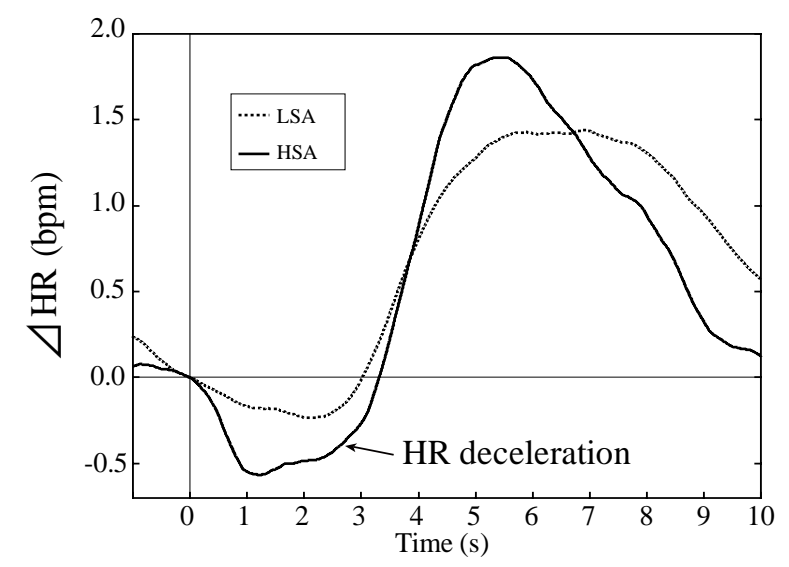

(a)

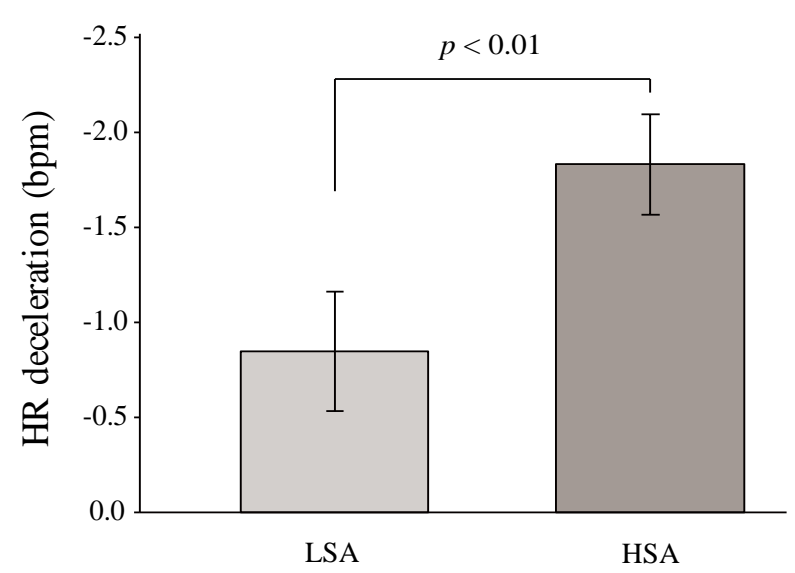

(b)

Figure 2. HR responses during the gaze detection experiment. (a) Mean HR (bpm) waveforms in response to stimuli in the two social anxiety groups. (b) Mean HR deceleration in the two social anxiety groups. 


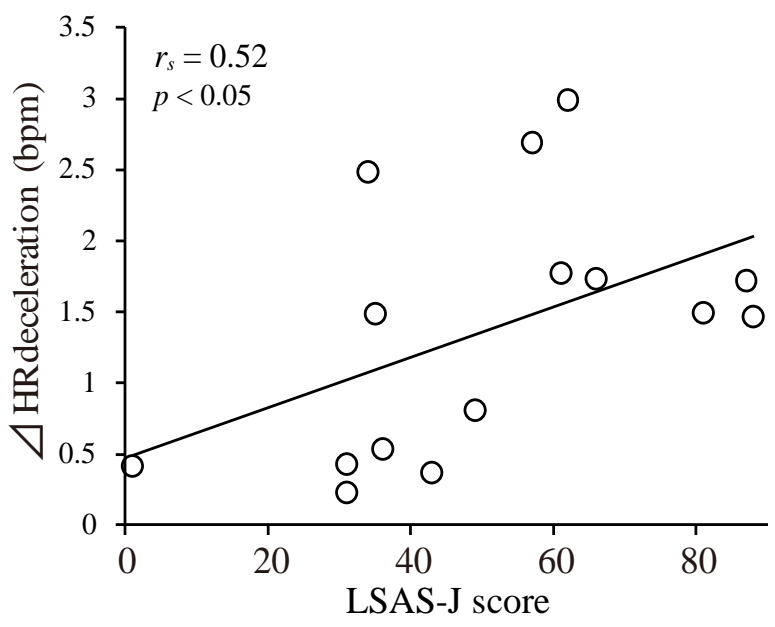

(a)

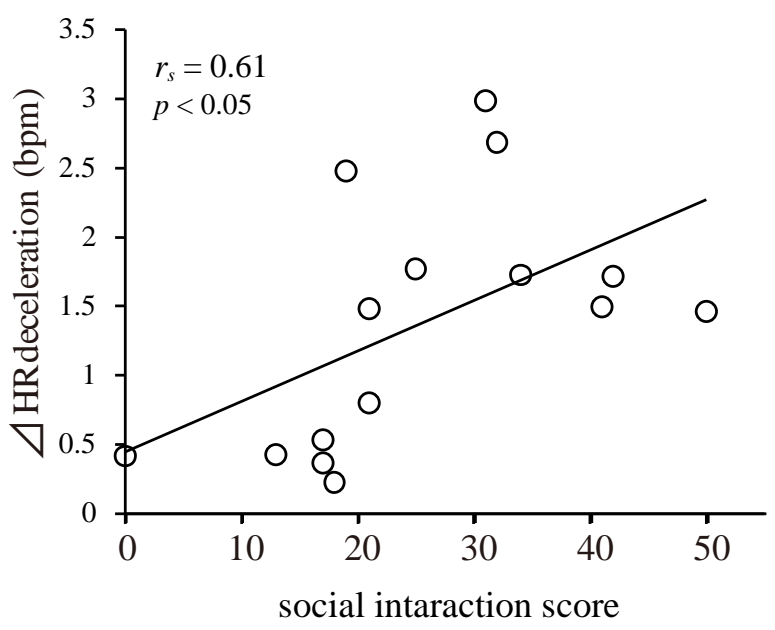

(b)

Figure 3. Correlations between LSAS-J score and HR deceleration. (a) Regression line for a positive correlation between HR deceleration and LSAS-J score. (b) Regression line for a positive correlation between HR deceleration and social interaction score. Spearman's rank correlation coefficients were $r_{s}=0.52(p<0.05)$ and $r_{s}=0.61(p<0.05)$ for the LSAS-J score and social interaction score, respectively.

addition, a positive correlation between social interaction score, which is a subscale of the LSAS-J, and HR deceleration was significant $\left(r_{s}=0.61, p<0.05\right.$, Figure 3$)$. We did not find any other significant correlations between LSAS-J and HR deceleration.

\section{Discussion}

In this study, we investigated the relationship between socially anxious tendencies and the autonomic reactions of participants while they completed a gaze detection task. Our results showed that HR deceleration in high social anxiety subjects was greater than that in low social anxiety subjects: the greater the level of social anxiety, the greater the HR deceleration. Therefore, a tendency towards social anxiety appears to affects autonomic responses during perception of eye gazes. In individuals with a high tendency towards social anxiety, the gazes of others may be processed as a threat, and thus may induce emotional stress.

Socially anxious individuals have been found to exhibit an attentional bias toward angry faces in visual attention paradigms (Horley, Williams, Gonsalvez, \& Gordon, 2004). Additionally, highly socially anxious individuals exhibit fear in response to neutral face (Wieser et al., 2009). Recent studies have shown that in individuals with SAD, self-directed gaze might elicit fears of being stared at (Horley et al., 2003; Moukheiber et al., 2010; Weeks et al., 2013; Schneier et al., 2011). These studies suggest that self-directed gaze may elicit fears of being stared at in individuals with social anxiety, as these individuals appear to have an excessive fear of scrutiny (Den Boer, 2000). Thus, we presumed that our experimental stimuli were perceived as threatening by individuals with socially anxious tendencies.

In animals, fight/flight and freezing behaviors, which often induce HR acceleration and HR deceleration, respectively, are known as acute stress responses. In human studies, freezing behavior is thought to be a defense response that occurs in the presence of a threat or an expectation of harm (Hagenaars, Oitzl, \& Roelofs, 2014). Bradley et al. (2001) argued that heart rate deceleration in participants when viewing an unpleasant picture from the IAPS is analogous to freezing behavior in animals when encountering a predator. They further suggested that human freezing behavior is part of a cognitive state in which the individual is orienting to the sensory input, processing contextual details, retrieving relevant information from memory, and implicitly preparing for a possible action. Several studies have reported strong HR deceleration when participants viewed pictures depicting a threat (e.g. injured human body) (Azevedo et al., 2005; Hermans et al., 2012). Therefore, we suggest that the acute stress response triggered by our experimental stimuli is freezing behavior, which often induces HR decelerations.

SAD is often accompanied by physiological symptoms associated with excessive autonomic nervous system activation, such as blushing, excessive sweating, and heart palpitations. In social anxiety research, cardiovascu- 
lar responses have been used as physiological measurements of anxiety. For example, individuals with SAD exhibited a greater degree of HR acceleration compared with healthy controls in a public speaking task (Beidel, Turner, \& Dancu, 1985). In an experiment conducted by Wieser, Pauli, Alpers, \& Mühlberger (2009), participants were instructed to fixate on the eye region of a neutral animated face until the eyes started to move. After movement onset, the participants were allowed to freely gaze around, avoiding the eye region if they preferred to do so. The researchers found HR acceleration in highly socially anxious participants while they observed direct gazes. As participants could escape from the threat, their defense strategy was active (fight/flight response), and so it is logical that their fight/flight response induced HR acceleration.

In contrast, HR deceleration had been observed when participants were instructed to fixate on unpleasant stimuli in an inescapable situation, such as the gaze stimuli in our experiment or unpleasant pictures from the IAPS used by Bradley et al. (2001). We suggest that participants who perceived the experimental stimulus as a threat experienced HR deceleration. Moreover, another study found that reduced body motion in response to a social threat (angry faces) was accompanied by HR deceleration and was significantly correlated with state anxiety score (Roelofs, Hagenaars, \& Stins, 2010). State anxiety score refers to how anxious a person is feeling at the time of a perceived threat (Spielberger \& Sydeman, 1994). Therefore, our results suggest that the gazes of others were perceived as a threat in individuals with socially anxious tendencies, and that HR deceleration during gaze perception can be an effective biological marker for studying social anxiety.

Finally, methodological limitation should be mentioned here. We measured HR deceleration and found that other's gaze would be perceived as a threat in highly socially anxious individuals. However, how central nervous system responds to gazes of others in individuals with socially anxious tendencies was not directly examined in the present study. A few studies have shown that negative facial expressions (angry or fear) induced hyper activation of amygdala in individuals with SAD (Blair et al., 2008; Labuschagne et al., 2010). Another study reported that highly socially anxious individuals elicited a greater front-central P2 during perception of negative facial expressions (Moser, Huppert, Duval, \& Simons, 2008). These findings in the neuroimaging literature can compensate our findings to further understand the abnormal process of gaze perception in socially anxious tendencies.

\section{Conclusion}

This study aims to reveal the effects of socially anxious tendencies on HR responses during gaze perception. The results suggest that the gazes of others are perceived as a threat and induce HR decelerations in individuals with socially anxious tendencies. The HR deceleration during gaze perception can be an effective biological marker for studying social anxiety.

\section{Acknowledgements}

This work was supported by Grants-in-Aid for Scientific Research (25700015 and 26119529) from the Japan Society for the Promotion of Science (JSPS).

\section{References}

American Psychiatric Association (2013). Diagnostic and Statistical Manual of Mental Disorders (5th ed.). Arlington, VA: American Psychiatric Publishing.

Azevedo, T. M., Volchan, E., Imbiriba, L. A., Rodrigues, E. C., Oliveira, J. M., Oliveira, L. F., \& Vargas, C. D. (2005). A Freezing-Like Posture to Pictures of Mutilation. Psychophysiology, 42, 255-260. http://dx.doi.org/10.1111/j.1469-8986.2005.00287.x

Baron-Cohen, S. (1995). Theory of Mind and Face-Processing: How Do They Interact in Development and Psychopathology? In D. Cicchetti, \& Cohen D. J. (Eds.), Developmental Psychopathology, Volume 1, Theory and Method (pp. 343-356). New York, NY: John Wiley \& Sons.

Beck, A. T., Steer, R. A., Ball, R., \& Ranieri, W. (1996). Comparison of Beck Depression Inventories-IA and -II in Psychiatric Outpatients. Journal of Personality Assessment, 67, 588-597. http://dx.doi.org/10.1207/s15327752jpa6703_13

Beidel, D. C., Turner, S. M., \& Dancu, C. V. (1985). Physiological, Cognitive and Behavioral Aspects of Social Anxiety. Behaviour Research and Therapy, 23, 109-117. http://dx.doi.org/10.1016/0005-7967(85)90019-1

Blair, K., Shaywitz, J., Smith, B. W., Rhodes, R., Geraci, M., Jones, M., \& Pine, D. S. (2008). Response to Emotional Expressions in Generalized Social Phobia and Generalized Anxiety Disorder: Evidence for Separate Disorders. The Ameri- 
can Journal of Psychiatry, 165, 1193-1202. http://dx.doi.org/10.1176/appi.ajp.2008.07071060

Bradley, M. M., Codispoti, M., Cuthbert, B. N., \& Lang, P. J. (2001). Emotion and Motivation I: Defensive and Appetitive Reactions in Picture Processing. Emotion, 1, 276-298. http://dx.doi.org/10.1037//1528-3542.1.3.276

Cannon, W. B. (1915). Bodily Changes in Pain, Hunger, Fear and Rage: An Account of Recent Researches into the Function of Emotional Excitement. New York, NY: D Appleton \& Company. http://dx.doi.org/10.7326/0003-4819-39-2-383_1

Den Boer, J. A. (2000). Social Anxiety Disorder/Social Phobia: Epidemiology, Diagnosis, Neurobiology, and Treatment. Comprehensive Psychiatry, 41, 405-415. http://dx.doi.org/10.1053/comp.2000.16564

Greist, J. H. (1995). The Diagnosis of Social Phobia. Journal of Clinical Psychiatry, 56, 5-12. http://dx.doi.org/10.1177/0145445500241006

Hagenaars, M. A, Oitzl, M., \& Roelofs, K. (2014). Updating Freeze: Aligning Animal and Human Research. Neuroscience and Biobehavioral Reviews, 47C, 165-176. http://dx.doi.org/10.1016/j.neubiorev.2014.07.021

Henry, J. P., \& Stephens, P. M. (1977). Stress, Health, and the Social Environment (1st ed.). New York, NY: Springer New York. http://dx.doi.org/10.1007/978-1-4612-6363-0

Hermans, E. J., Henckens, M. J. A. G., Roelofs, K., \& Fernández, G. (2012). Fear Bradycardia and Activation of the Human Periaqueductal Grey. NeuroImage, 66, 278-287. http://dx.doi.org/10.1016/j.neuroimage.2012.10.063

Horley, K., Williams, L. M., Gonsalvez, C., \& Gordon, E. (2003). Social Phobics Do Not See Eye to Eye: A Visual Scanpath Study of Emotional Expression Processing. Journal of Anxiety Disorders, 17, 33-44. http://dx.doi.org/10.1016/S0887-6185(02)00180-9

Horley, K., Williams, L. M., Gonsalvez, C., \& Gordon, E. (2004). Face to Face: Visual Scanpath Evidence for Abnormal Processing of Facial Expressions in Social Phobia. Psychiatry Research, 127, 43-53. http://dx.doi.org/10.1016/j.psychres.2004.02.016

Kessler, R. C., Petukhova, M., Sampson, N. A., Zaslavsky, A. M., \& Wittchen, H. U. (2012). Twelve-Month and Lifetime Prevalence and Lifetime Morbid Risk of Anxiety and Mood Disorders in the United States. International Journal of Methods in Psychiatric Research, 21, 169-184. http://dx.doi.org/10.1002/mpr.1359

Kessler, R. C., Stang, P., Wittchen, H. U., Stein, M., \& Walters, E. E. (1999). Lifetime Co-Morbidities between Social Phobia and Mood Disorders in the US National Comorbidity Survey. Psychological Medicine, 29, 555-567. http://dx.doi.org/10.1017/S0033291799008375

Labuschagne, I., Phan, K. L., Wood, A., Angstadt, M., Chua, P., Heinrichs, M., \& Nathan, P. J. (2010). Oxytocin Attenuates Amygdala Reactivity to Fear in Generalized Social Anxiety Disorder. Neuropsychopharmacology: Official Publication of the American College of Neuropsychopharmacology, 35, 2403-2413. http://dx.doi.org/10.1038/npp.2010.123

Liebowitz, M. R. (1987). Social Phobia. Modern Problems of Pharmacopsychiatry, 22, 141-173. http://dx.doi.org/10.1159/000414022

Moser, J. S., Huppert, J. D., Duval, E., \& Simons, R. F. (2008). Face Processing Biases in Social Anxiety: An Electrophysiological Study. Biological Psychology, 78, 93-103. http://dx.doi.org/10.1016/j.biopsycho.2008.01.005

Moukheiber, A., Rautureau, G., Perez-Diaz, F., Soussignan, R., Dubal, S., Jouvent, R., \& Pelissolo, A. (2010). Gaze Avoidance in Social Phobia: Objective Measure and Correlates. Behaviour Research and Therapy, 48, 147-151. http://dx.doi.org/10.1016/j.brat.2009.09.012

Roelofs, K., Hagenaars, M. A., \& Stins, J. (2010). Facing Freeze: Social Threat Induces Bodily Freeze in Humans. Psychological Science: A Journal of the American Psychological Society/APS, 21, 1575-1581. http://dx.doi.org/10.1177/0956797610384746

Schneier, F. R., Rodebaugh, T. L., Blanco, C., Lewin, H., \& Liebowitz, M. R. (2011). Fear and Avoidance of Eye Contact in Social Anxiety Disorder. Comprehensive Psychiatry, 52, 81-7. http://dx.doi.org/10.1016/j.comppsych.2010.04.006

Spielberger, C. D., \& Sydeman, S. J. (1994). State-Trait Anxiety Inventory and State-Trait Anger Expression Inventory. In M. E. Maruish (Ed.), The Use of Psychological Testing for Treatment Planning and Outcome Assessment (pp. 292-321). Hillsdale, NJ, USA: L. Erlbaum Associates. http://dx.doi.org/10.1002/9780470479216.corpsy0943

Weeks, J. W., Howell, A. N., \& Goldin, P. R. (2013). Gaze Avoidance in Social Anxiety Disorder. Depression and Anxiety, 30, 749-756. http://dx.doi.org/10.1002/da.22146

Wieser, M. J., Pauli, P., Alpers, G. W., \& Mühlberger, A. (2009). Is Eye to Eye Contact Really Threatening and Avoided in Social Anxiety?-An Eye-Tracking and Psychophysiology Study. Journal of Anxiety Disorders, 23, 93-103. http://dx.doi.org/10.1016/j.janxdis.2008.04.004 